\title{
Rotating Machine Fault Diagnosis Based on Optimal Morphological Filter and Local Tangent Space Alignment
}

\author{
Shaojiang Dong, ${ }^{1}$ Lili Chen, ${ }^{1}$ Baoping Tang, ${ }^{2}$ Xiangyang Xu, \\ Zhengyuan Gao, ${ }^{3}$ and Juan Liu ${ }^{4}$ \\ ${ }^{1}$ School of Mechatronics and Automotive Engineering, Chongqing Jiaotong University, Chongqing 400074, China \\ ${ }^{2}$ The State Key Laboratory of Mechanical Transmission, Chongqing University, Chongqing 400030, China \\ ${ }^{3}$ Chongqing Academy of Metrology and Quality Inspection, Chongqing 401123, China \\ ${ }^{4}$ Chongqing University of Education, Chongqing 400065, China
}

Correspondence should be addressed to Lili Chen; 1016694222@qq.com

Received 4 October 2014; Revised 20 November 2014; Accepted 28 November 2014

Academic Editor: Alicia Gonzalez-Buelga

Copyright (c) 2015 Shaojiang Dong et al. This is an open access article distributed under the Creative Commons Attribution License, which permits unrestricted use, distribution, and reproduction in any medium, provided the original work is properly cited.

\begin{abstract}
In order to identify the fault of rotating machine effectively, a new method based on the morphological filter optimized by particle swarm optimization algorithm (PSO) and the nonlinear manifold learning algorithm local tangent space alignment (LTSA) is proposed. Firstly, the signal is purified by the morphological filter; the filter's structure element (SE) is selected by PSO method. Then the filtered signals are decomposed by the empirical mode decomposition (EMD) method, and the extract features are mapped into the LTSA to extract the character features; then the support vector machine (SVM) model is used to achieve the rotating machine fault diagnosis. The proposed method is evaluated by vibration signals measured from bearings with faults. Results show that the method can effectively remove the noise and extract the fault features, so the rotating machine fault diagnosis can be achieved effectively.
\end{abstract}

\section{Introduction}

Rotating machinery is widely used in industry. Unexpected machine faults could cause unscheduled downtime and loss. So it is very important to diagnose the fault of the rotating machinery [1]. To diagnose the fault, we should take three main steps: (1) the collection of the fault signals of the machine, (2) the extraction of the fault features though signal processing methods, and (3) condition identification and fault diagnosis. In this research, we choose the vibration signals to identify the faults. However, the vibration signals, collected by a sensor, are often severely polluted by various noises, for example, the background noise presented in the measurement device, and the interfering vibrations generated by other mechanical components which are of no significance for condition monitoring [2]. The noise can seriously affect the results, so we need to remove it. There are many methods for filtering the noise, but they have different shortcomings [3]. This paper uses the morphological filter to remove the noise [4]. It can decompose the original signal into several physical parts according to certain geometric characteristics. The people who first introduced morphological filter into fault diagnosis of rotating machinery were Nishida et al. [5]. To construct the filter, we should first select the filter's structure element (SE); SE can decide the shape of the filter and determine the performance of morphological filter, so many researches had been done to select the optimal SE. In this paper, the SE is chosen through PSO algorithm to optimize the SE and construct an effective morphological filter.

After removing the noise, the EMD method is used to decompose the signal and the Shannon entropy of the intrinsic mode function (IMF) is used to extract the features; however, the extracted features remained high-dimensional and has the characteristic of nonlinear, so we need to reduce the dimensionality in order to extract the feature and diagnose the fault of the rotating machine [6]. In this paper a new fault feature extraction method LTSA [7] is used. 
Through LTSA we can observe high-dimensional nonlinear data sets directly [8]. It can be used as a preprocessing method to reduce high-dimensional data into more easily handled low-dimensional data, so it has been used in many fields, such as face recognition, character recognition, and image recognition [9]. After the features are treated by the LTSA, the SVM model [10] is used to achieve the bearing fault diagnosis.

The rest of this paper is organized as follows. In Section 1, the morphological filter is described and the character of it is discussed. The PSO algorithm is proposed to optimize the SE. In Section 2, the theory of LTSA is described. In Section 3, the proposed method is validated by practical examples. Then, we conclude this work in Section 4.

\section{Optimized Morphological Filter for Noise Removing}

The main characteristic of morphological filter is that it can keep the phase and amplitude while filtering the signal; the whole work is done in the time domain and is compared with other methods such as Fourier transform and the wavelet transform; this calculation is simpler [11]. To deal with the signal only the calculation of adding and subtracting is done. The performance of the transformation is decided by the SE and the corresponding transformation operations.

2.1. The Fundamental Theory of Morphological Filter. Morphological filter with functional SE for one-dimensional time series data was first presented by Maragos and Schafer [12] in 1987; it requires less computational time than other traditional filters. Through constantly moving the SE to match the signal, the purposes of denoising can be achieved. The transformation consists of four basic operations: erosion, dilation, opening, and closing operation. The operations between two sets $A$ and $B$ of numbers are defined as follows:

$$
\begin{aligned}
& A \oplus B=\left\{z \in Z^{n}: z=a+b, a \in A, b \in B\right\}=\bigcup_{b \in B}(A)_{b}, \\
& A \Theta B=\left\{z \in Z^{n}: z=a-b, a \in A, b \in B\right\}=\bigcap_{b \in B}(A)_{-b} .
\end{aligned}
$$

In formula, $\oplus, \Theta$ symbols correspond to the dilation and erosion operations. Erosion means to filter the noise in the internal of the signal. The dilation has the role of extending the signal. The opening and closing operations are defined as follows:

$$
\begin{aligned}
& A \circ B=(A \Theta B) \oplus B, \\
& A \cdot B=(A \oplus B) \Theta B .
\end{aligned}
$$

In formula, o, - symbols correspond to the opening operation and closing operation. But the erosion operation can remove all structures that do not accommodate with the SE and can cause the contraction of the other structures which may contain useful information. So the operation of dilation can resume those structures which contain useful information.
2.2. Design of Morphology Filter. Supposing the original signal $f(x)$ is a discrete function defined on $D_{f}$ boundary and the structural element $g(x)$ is a discrete function defined on $D_{g}$ boundary, $y$ form of open-closing and close-opening filters is

$$
\begin{aligned}
& F_{\mathrm{OC}}(f(x))=(f \circ g \circ g)(x), \\
& F_{\mathrm{CO}}(f(x))=(f \circ g \circ g)(x) .
\end{aligned}
$$

The morphological filter removes the positive and negative noise by constructing a form of open-closing and closeopening operation. But the opening operation leads to the contraction of the output of the open-closing operation and the closing operation leads to the expansion of the output of the close-opening operation, so there is something wrong with the value, which directly affect the denoising effort of the filter. In order to avoid this problem, we construct the nonlinear filter in the following form:

$$
y(x)=\frac{1}{2}\left[F_{\mathrm{CO}}(f(x))+F_{\mathrm{OC}}(f(x))\right] .
$$

In this research, the PSO algorithm is used to choose the amplitudes of the SEs.

There are various kinds of SEs, such as flat SE, triangular SE, and semicircular SE [13]. The size of the SE relies on the period length of the noise, so the size of the SE is chosen from 3 to $(2 * L / 2+1)$, where $L$ is the estimated length of the noise period [4].

2.3. The Optimization of Morphological Filter. Now there are many optimal algorithms, for example, genetic algorithm (GA), ant colony algorithm, particle swarm optimization algorithm (PSO), and so on. PSO is a population based algorithm developed in 1995 [14, 15]; it is based on the theory of swarm intelligence which is similar to GA but it searches the optimal particle in the whole space and does not achieve optimization through evolution. Compared with the GA and ant colony, the PSO is easy to implement and is an effective global optimization algorithm. PSO searches the space in the $n$-dimension through the change of the position and the speed to find the optimal solution of the current population. Therefore, we choose the PSO to optimize the SE of the morphological filter. Each individual (particle) adjusts its flight speed and position according to the following formula:

$$
\begin{gathered}
v_{i j}(t+1)=w v_{i j}(t)+c_{1} r_{1 j}\left(p_{i j}(t)-x_{i j}(t)\right) \\
+c_{2} r_{2 j}\left(p_{i j}(t)-x_{i j}(t)\right) \\
x_{i j}(t+1)=x_{i j}(t)+v_{i j}(t+1)
\end{gathered}
$$

where " $j$ " represents the $j$-dimension. The subscript " $i$ " represents the $i$-dimension. The subscript " $t$ " represents the $t$ generation. The $w$ represents the weight which has a great influence on the performance of optimizing, the larger value of the $w$ can help the PSO avoid the local minimum value but may cause local oscillator, and the smaller value of the $w$ can help the PSO concentrate accurately but may be too slow to 
achieve the result. So we change the way to construct the $w$ as follows:

$$
w=w_{\max }-t \times \frac{w_{\max }-w_{\min }}{t_{\max }}
$$

where $w_{\max }, w_{\min }$, respectively, represent the maximum and minimum inertia weight.

$c_{1}, c_{2}$ are learning factors; the $c_{1}$ represents the best flight speed in the memory location. The $c_{2}$ represents the best position of particle groups in the memory. $r_{1} \sim U(0,1)$, $r_{2} \sim U(0,1)$ represent two independent random functions.

2.4. Determination of the Fitness Function. In order to obtain the optimized SE, a criterion should be built as the fitness function of the PSO. There is some relationship between the fitness function and the signal. The fitness function should be selected according to the characteristics of the fault signal and this process needs the criteria to be changed according to the optimization process. Therefore, the signal-to-noise ratio (SNR) is selected as the fitness function. The function is defined in the following form:

$$
\begin{gathered}
R_{\mathrm{SN}}(x)=10 \lg \frac{p(w)}{N(w)}, \\
p(w)=|Y(w)|^{2}
\end{gathered}
$$

where $R_{\mathrm{SN}}(x)$ is the SNR function of the system, $y(x)$ is the output of the filter, $Y(w)$ is the power spectrum of the output signal, and $N(w)$ is the power spectrum of the noise signal.

The process of optimizing the SE based on the PSO is given as follows.

(1) At the beginning of the optimization process, randomly initialize positions and velocities, pbest, gbest of the particles.

(2) Set the parameters of SEs equal to the parameters of the particles' positions.

(3) Use the SEs to construct the morphological filters.

(4) Use the morphological filters to remove the noise and get the filtered signal $y(x)=(1 / 2)\left[F_{\mathrm{CO}}(f(x))+\right.$ $\left.F_{\mathrm{OC}}(f(x))\right]$; then use (7) to calculate the current fitness value $R\left(x_{i}\right)$ of each particle.

(5) Use the pbest to construct the morphological filter, calculate the fitness value $R\left(x_{p}\right)$, and compare the $R\left(x_{p}\right)$ with $R\left(x_{i}\right)$. If $R\left(x_{i}\right)$ is greater than $R\left(x_{p}\right)$, set the parameters of particle $x_{i}$ to the pbest.

(6) Use the gbest to construct the morphological filter, calculate the fitness value $R\left(x_{g}\right)$, and compare the $R\left(x_{p}\right)$ with $R\left(x_{g}\right)$. If $R\left(x_{p}\right)$ is greater than $R\left(x_{g}\right)$, set the parameters of pbest to the gbest.

(7) For each particle $i$ in the swarm, calculate positions $x_{i+1}$, velocities $V_{i+1}$ using (5).

(8) If the termination conditions have not been met, return to Step (3). Otherwise, end the loop.
2.5. Comparison among Different Methods. To evaluate the denoising efficiency of different methods, the comparison between the optimal morphological filter and other filtering techniques (i.e., the morphological filter of flat SE and the wavelet of Morlet) is carried out. The simulated signal is formulated as follows (the sampling frequency is $1000 \mathrm{~Hz}$ and the sampling time is $1 \mathrm{~s})$ :

$$
x(t)=x_{1}(t)+2 x_{2}(t)+x_{3}(t)
$$

where $x_{1}(t)$ is the sum of three harmonic waves $x_{1}(t)=$ $0.8 \sin (2 \pi \cdot 10 t)+\sin (2 \pi \cdot 30 t)+0.3 \sin (2 \pi \cdot 45 t), x_{2}(t)$ is $\sin (2 \pi t)$, and $x_{3}(t)$ is the Gaussian noise $(\mathrm{SNR}=16.54 \mathrm{~dB})$.

We use the following three methods to filter the signal: the optimal filter (the optimal size of the SE is 3, and the optimal amplitudes of the SE are $(0.0032,0.0151,0.0031)$ ); the morphological filter of flat SE (the size of the SE is 3, and the amplitudes of the SE are $(0,0,0))$; and the wavelet of Morlet (the optimal parameters $\beta=0.5$ and $f_{0}=1 \mathrm{~Hz}$ ). Among them, the parameters of the PSO for optimizing the SE are set as the original position $(0,0,0)$ and velocity $(0,0,0)$, pbest $(0,0,0)$, gbest $(0,0,0)$, the population scale $m=20$, the terminal interaction time $t_{\max }=300$, the inertia weight $w=0.5, c_{1}=c_{2}=1.2$, and the search space dimension $d=3$. The obtained results are shown in Figure 1.

From the results we can conclude that the optimal morphological filter effectively extract the impulse signal and remove the noise. In contrast, the morphological filter of flat SE and the Morlet wavelet can eliminate most of the noise, but there is still a certain noise reserved. Therefore, we choose the optimal morphological filter to remove the noise.

\section{Basic Concepts of Local Tangent Space Alignment}

The basic idea of LTSA is to use the tangent space of sample points to represent the geometry of the local character. Then these local manifold structures of space are lined up to construct the global coordinates. Given a data set $X=\left[x_{1}, x_{2}, \ldots, x_{N}\right], x_{i} \in R^{m}$, a mainstream shape of $d$ dimension $(m>d)$ is extracted. The LTSA feature extraction algorithm is as follows:

(1) extracting local information: for each $x_{i}, i=$ $1,2, \ldots, N$, the Euclidean distance to determine a set $x_{i}=\left[x_{i, 1}, x_{i, 2}, \ldots, x_{i, k_{i}}\right]$ of its neighborhood adjacent points ( $k$ nearest neighbors, e.g.) is used;

(2) local linear fitting: in the neighborhood of data points $x_{i}$, a set of orthogonal basis $Q_{i}$ can be selected to construct the $d$-dimension neighborhood space of $x_{i}$ and the orthogonal projection of each point $x_{i, j}(j=$ $1,2, \ldots, N)$ can be calculated to the tangent space of $\theta_{j}^{(i)}=Q_{i}^{T}\left(x_{i, j}-\overline{x_{i}}\right) ; \overline{x_{i}}$ is the mean data for the neighborhood; the orthogonal projection in the tangent space of neighborhood data of $x_{i}$ is composed of local coordinate $\Theta_{i}=\left[\theta_{(i), 1}, \theta_{(i), 2}, \ldots, \theta_{(i), k_{i}}\right]$ that describes the most important information of the geometry of the $x_{i}$; 


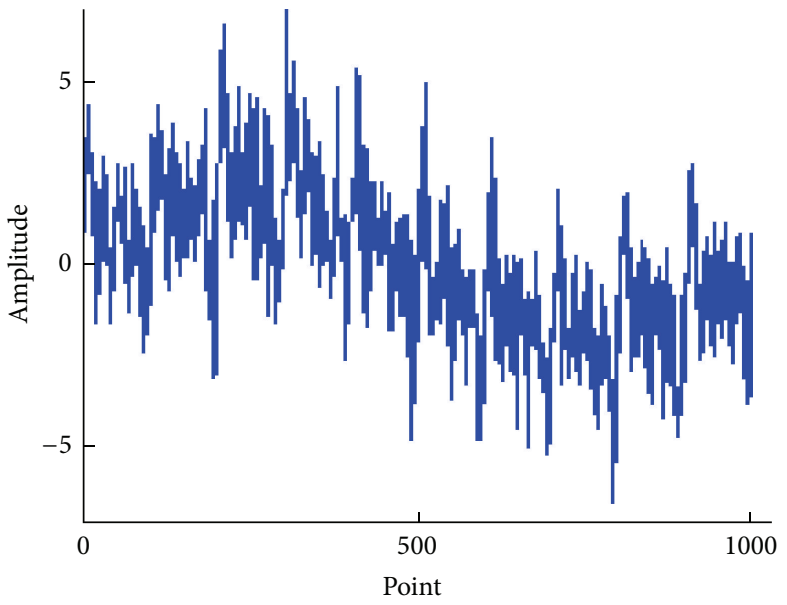

(a)

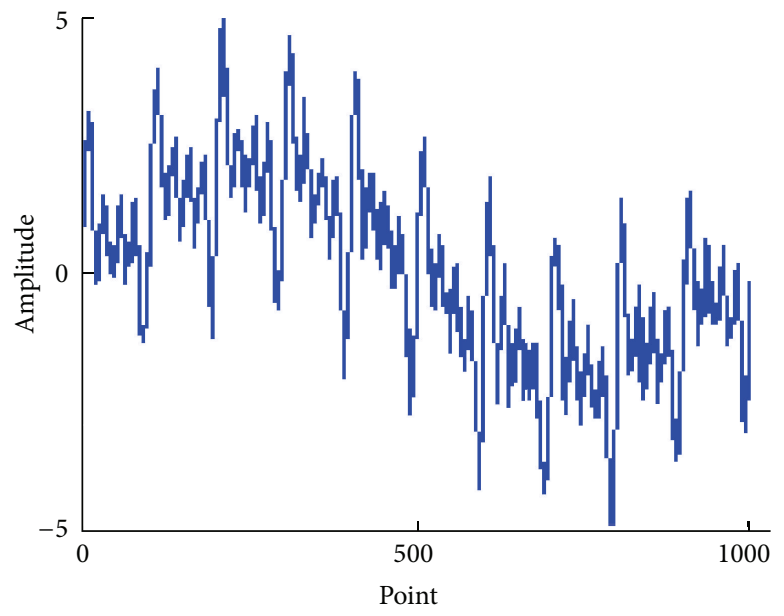

(c)

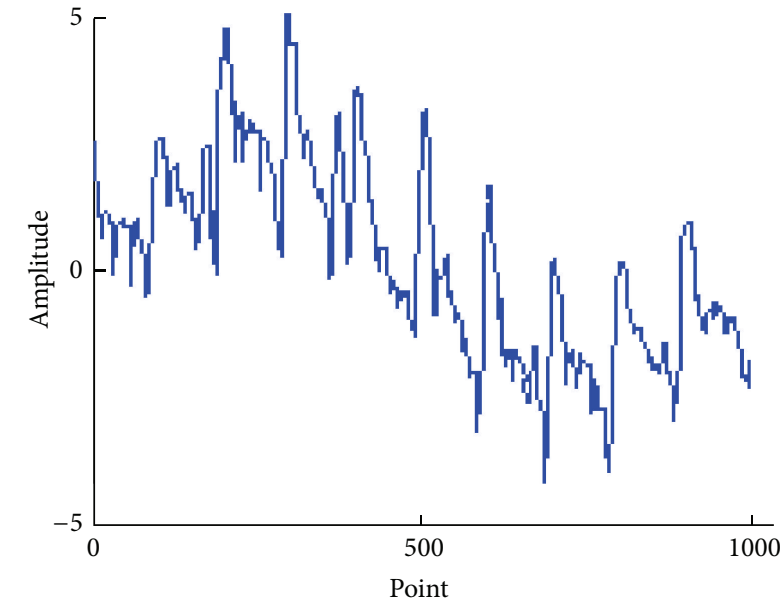

(b)

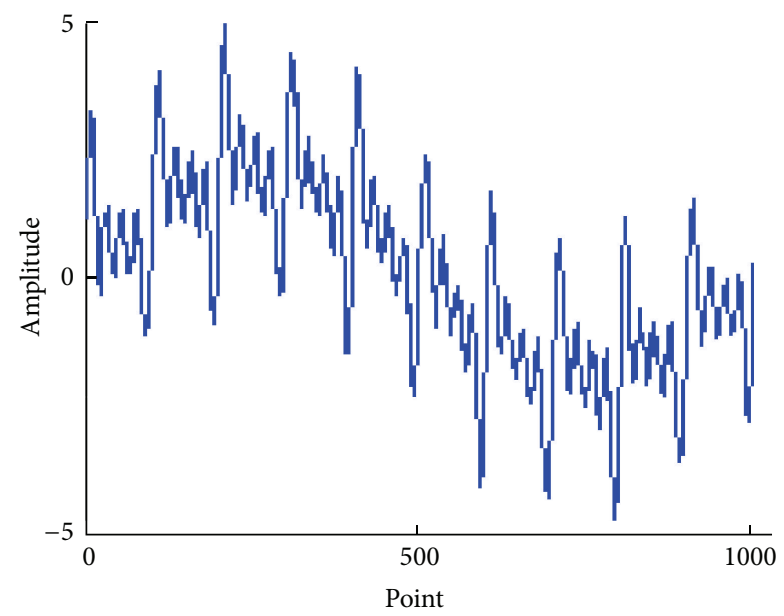

(d)

FIGURE 1: Comparison of denoised results obtained by optimal filter, morphological filter of flat SE, and the wavelet of Morlet, for the impulse signal: (a) the original signal, (b) the wavelet of Morlet, (c) the morphological filter of flat SE, and (d) the optimized morphological filter.

(3) global order of the local coordinates: supposing the global coordinates of $x_{i}$ converted by the $\Theta_{i}$ is $T_{i}=$ $\left[t_{i 1}, t_{i 2}, \ldots, t_{i k_{i}}\right]$, then the error is

$$
E_{i}=T_{i}\left[I-\left(\frac{1}{k}\right) e e^{T}\right]-L_{i} \Theta_{i}
$$

where the $I$ is the identity matrix, the $e$ is the unit vector, the $k$ is the points number of the neighborhood, and the $L_{i}$ is the transformation matrix; in order to minimize the error, the $T_{i}$ and $L_{i}$ should be found; then

$$
\begin{gathered}
L_{i}=T_{i}\left(I-\left(\frac{1}{k}\right) e e^{T}\right) \Theta_{i}^{*}, \\
E_{i}=T_{i}\left(I-\left(\frac{1}{k}\right) e e^{T}\right)\left(I-\Theta_{i}^{*} \Theta_{i}\right),
\end{gathered}
$$

where the $\Theta_{i}^{*}$ is the Moor-Penrose generalized inverse of $\Theta_{i}$; supposing the

$$
B=P W W^{T} P^{T}
$$

let $P=\left[P_{1}, P_{2}, \ldots, P_{N}\right], T P_{i}=T_{i}, P_{i}$ is a selected matrix from $0-1$, and $T$ is global coordinates; their weight matrix

$$
\begin{gathered}
W=\operatorname{diag}\left(W_{1}, W_{2}, \ldots, W_{N}\right), \\
W_{i}=\left(I-\left(\frac{1}{k}\right) e e^{T}\right)\left(I-\Theta_{i}^{*} \Theta_{i}\right) ;
\end{gathered}
$$

the constraint is

$$
T T^{T}=I_{d}
$$

(4) extract of the low-dimensional manifolds feature: since the $e$ is the eigenvalue of matrix $B$, so the 
corresponding minimum eigenvectors matrix is composed of eigenvalue; Section 2 to section $d+1$ of matrix $B$ make of the $T$. $T$ is the global coordinate mapping in the Mainstream form of low-dimensional transformed from the nonlinear high-dimensional data set of $X$.

The method consists of four procedures sequentially: data processing and features extracting, merging of the original features, and constructing-training SVM model for fault diagnosis. The role of each procedure is explained as follows.

Step 1 (data processing and features extraction). The optimized morphological filter is used to filter the signal, and the EMD signal processing methods are used to extract the original features from the collected mass vibration data.

Step 2 (merging of the original features). The LTSA method is used to extract the typical features and reduce the dimension of the features. The extracted features are used for training the SVM model.

Step 3 (construct the SVM model). The SVM model is constructed. The rotating machine fault diagnosis is achieved.

The flowchart of the proposed method is shown in Figure 2.

\section{Experimental Validation}

The proposed method is applied to bearing fault signals obtained from the Case Western Reserve University [16]. The bearing type in the experiments is SKF 6205-2RS JEM. Experiments were conducted by using a $2 \mathrm{hp}$ reliance electric motor. Bearings were seeded with faults by using electrodischarge machining. Faults were 0.021 inches in diameter and 0.011 inches in depth and were introduced at the inner raceway, rolling element (i.e., ball), and outer raceway. Motor speeds is set to 1797 RPM. Data were collected at 12,000 samples/second.

The parameters of the PSO for optimizing the SE are set to the following: the original position $(0,0,0)$ and velocity $(0,0,0)$, pbest $(0,0,0)$, gbest $(0,0,0)$, the population scale $m=$ 30 , the terminal interaction time $t_{\max }=300$, the inertia weight $w=0.5, c_{1}=c_{2}=1.2$, and the search space dimension $d=3$. The curve of the PSO optimization process is shown in Figure 3.

The fault signals are mashed with a lot of impulse, random signals, and noise. In order to verify the denoising effect of the optimized filter, the fault signals of inner and outer rings were used to be denoised. Filtering results are shown in Figures 4 and 5.

From the results we can get that Morlet wavelet can filter out some noise, but the effect is not very conspicuous; there is still some noise in the signal. The effect of the morphological filter of flat SE for denoising is better than the wavelet of Morlet. The optimal filter works very well in denoising and effectively shows the impact within and outside the bearing.

After the fault signals are filtered, 40 specimens are collected from each type of the three different faults; every

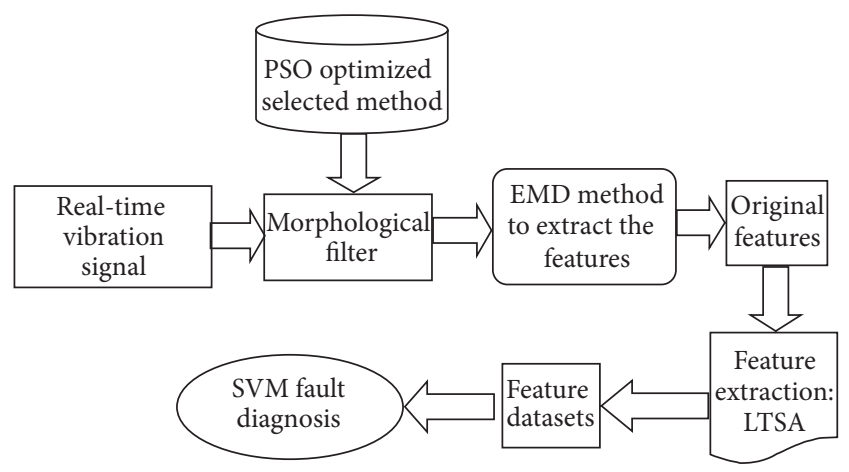

FIgURE 2: The flowchart of the proposed method.

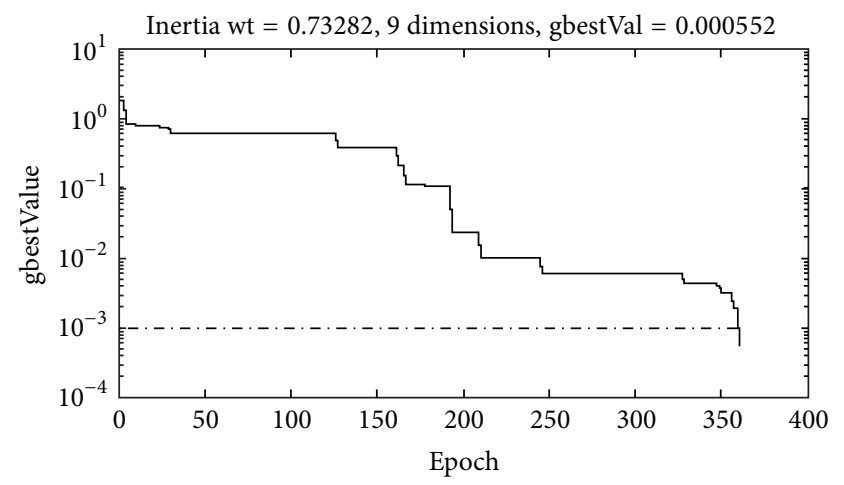

FIgURE 3: The curve of the PSO optimization process.

specimen has 1024 points. The EMD method is used to decompose the filtered signals and the Shannon entropy of the IMFs works as the original features.

The LTSA algorithm is used to deal with the original features. It switches the features from high-dimensional to the low-dimensional space to construct the liner onedimension characteristic curves of the faults. In this paper the parameters of the neighborhood factor $k$ equal 10 and the embedding dimension $d$ equals 3 . To demonstrate the superiority of the LTSA in this paper, the PCA was used. Figure 6 illustrated the result of the signal not purified by morphological filter and use of the LTSA to extract the original features and Figure 7 illustrated the result of the purified signal and use of the PCA to extract the original features, while using the purified signal and the LTSA to extract the original features in Figure 8.

It can be seen from Figure 6 that because of the effect of the noise, the LTSA analysis result is not very good; it cannot extract the typical features of the faults effectively. So it is necessary to remove the noise firstly.

It can be seen from Figure 7 that the effect of clustering the data types based on the purified signal and the PCA analysis is also not very good. The main reason is that the PCA analysis is mainly for the linear data; while dealing with the nonlinear data, it cannot work effectively.

From Figure 8, we can get that the combining of the purified signal and the LTSA can work well in clustering the data types and extracting the features effectively. 


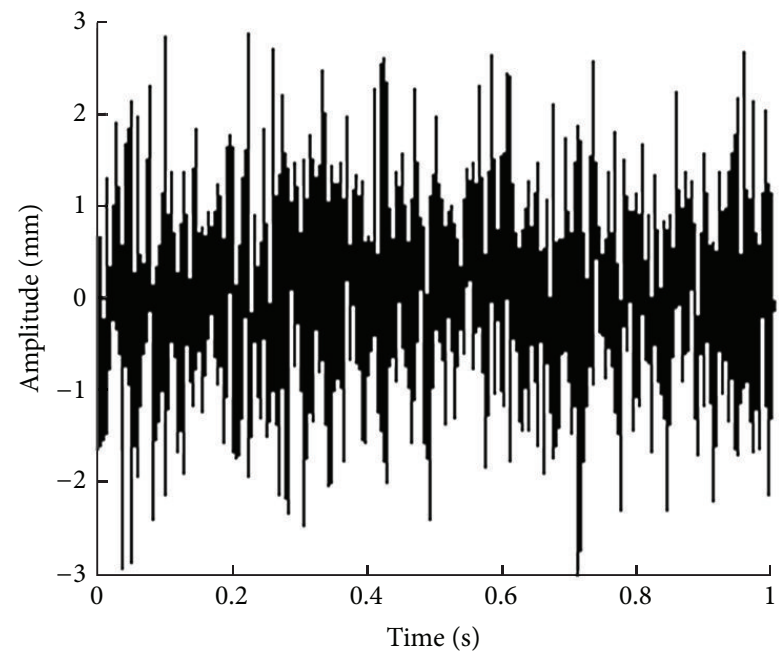

(a)

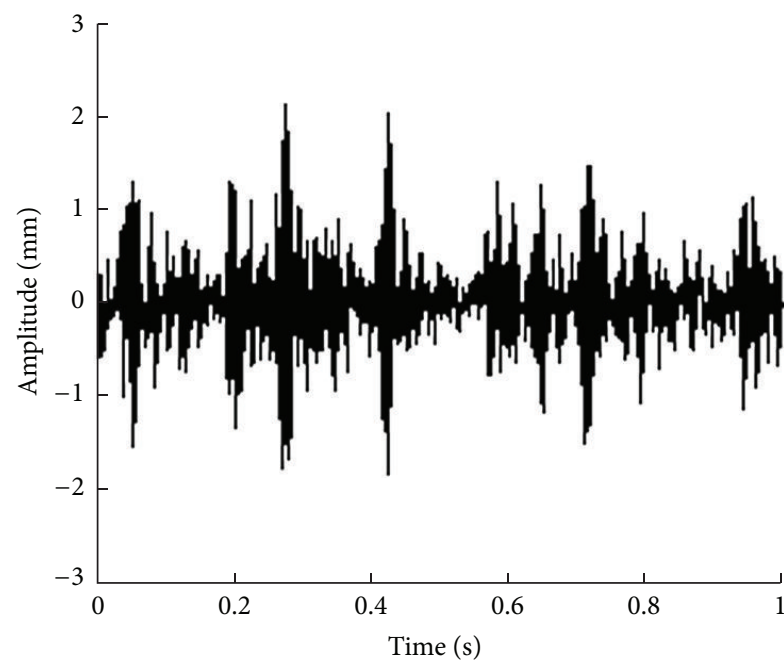

(c)

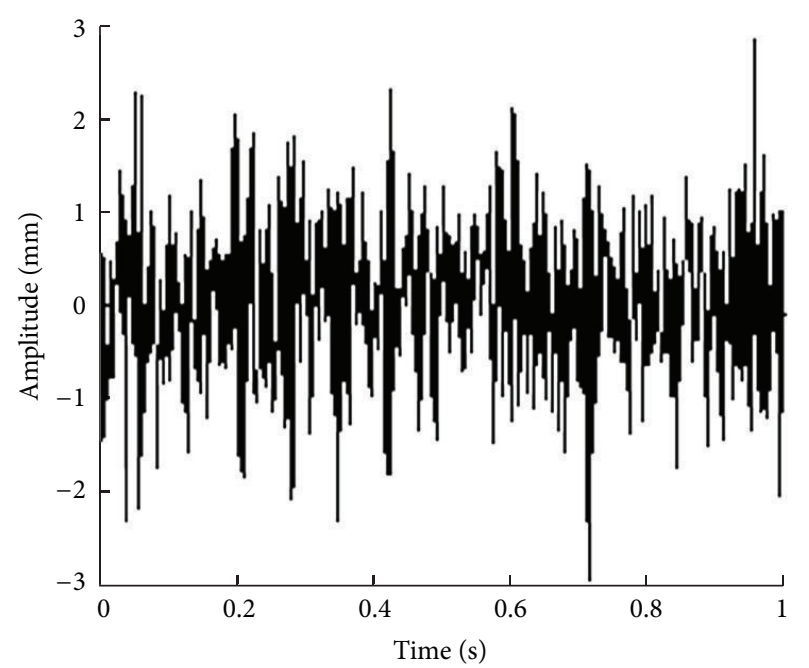

(b)

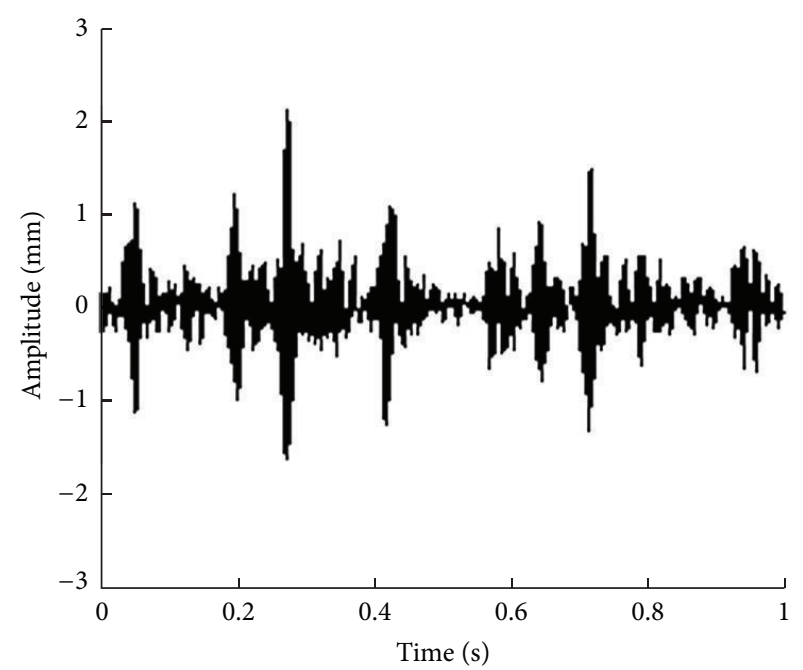

(d)

FIGURE 4: Comparison of denoising results obtained by the optimal filter $(0,0.01,0)$, the morphological filter of flat SE $(0,0$, 0$)$, and the wavelet of Morlet (with $w_{0}$ set to 5, $a_{j}$ equal to $a$ set to 0.3 ), for the vibration signal of a roller bearing with an inner-race fault: (a) the original signal, (b) the wavelet of Morlet, (c) the morphological filter of flat SE, and (d) the optimal filter.

Then the extracted features are input into the SVM to train the model and recognize the fault states (with penalty factor $C$ set to 100 and nuclear parameter $\gamma$ is set to 0.1 ) and the BP neural network (with the learning rate of the neural network 0.01; the iteration number is 2000; the training error is 0.001 ; the hidden number $n=15$ ). The comparison results are shown in Table 1.

From Table 1 we can see that the SVM can better identify and approach the sensitive features. So the choice of SVM to determine the bearing running states can effectively improve the recognition accuracy.

A comparison between the training and test time loss of different methods is implemented.

(1) The vibration data is filtered by the optimized morphological filter, and the EMD Shannon entropy is used to extract the typical features, the extract features
TABLE 1: The recognition rate of traditional RBF SVM and the MWSVM.

\begin{tabular}{lccc}
\hline \multirow{2}{*}{ Model type } & \multicolumn{3}{c}{ Recognition rate $\eta / \%$} \\
& Inner race fault & Ball fault & Outer race fault \\
\hline BP kernel & 95 & 90 & 93 \\
SVM & 100 & 100 & 100 \\
\hline
\end{tabular}

are direct input into the SVM, without the LTSA dimension reduction.

(2) The vibration data is filtered by the optimized morphological filter, and the EMD Shannon entropy is used to extract the typical features; the features are processed by LPP to reduce the dimension; then 


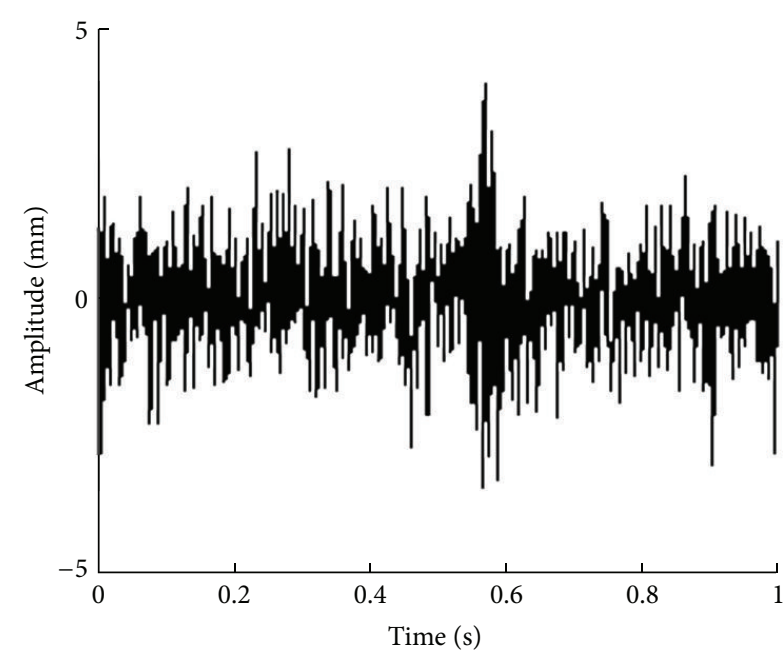

(a)

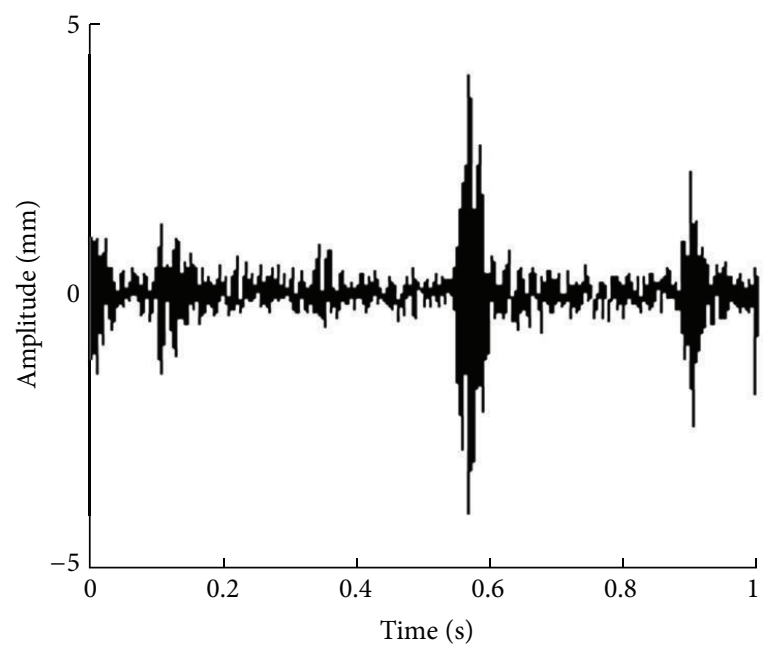

(c)

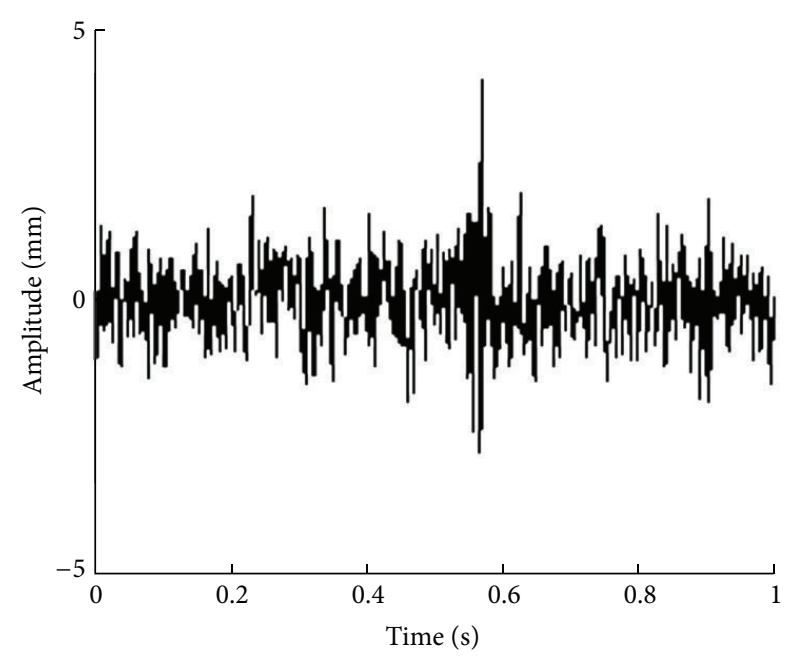

(b)

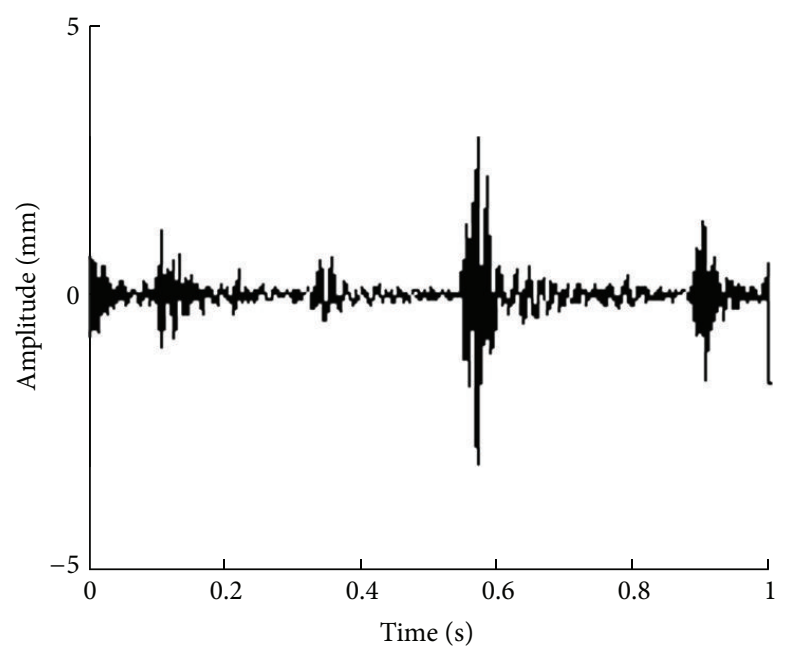

(d)

FiguRE 5: Comparison of denoising results obtained by the optimal filter, the morphological filter of flat SE, and the wavelet of Morlet, for the vibration signal of a roller bearing with an outer-race fault: (a) the original signal, (b) the wavelet of Morlet, (c) the morphological filter of flat SE, and (d) the optimal filter.

the extracted features are input into the BP neural network.

(3) The proposed method in this research.

The comparison results are shown in Table 2.

From Table 2 we can see that, after the dimension reduction, the recognition speed of SVM improved significantly. The time loss of the proposed method is the shortest; the reason is that the proposed method uses the LTSA to extract the typical features to make sure the SVM can efficiently deal with the fault.

\section{Conclusion}

(1) This research proposed uses the optimized morphological filter to purify the signal, so as to extract the
TABLE 2: The time loss of three different methods.

\begin{tabular}{lccc}
\hline Methods & $\begin{array}{c}\text { Without LTSA } \\
\text { dimension } \\
\text { reduction }\end{array}$ & $\begin{array}{c}\text { By BP neural } \\
\text { network }\end{array}$ & $\begin{array}{c}\text { The proposed } \\
\text { method }\end{array}$ \\
\hline Time/s & 231 & 215 & 210 \\
\hline
\end{tabular}

character features effectively from background noise more than other methods.

(2) The manifold learning method LTSA is used to reduce the dimension and data redundancy of the entropy features.

(3) In order to more accurately identify the bearing running state, the SVM is used to achieve the bearing fault diagnosis. 


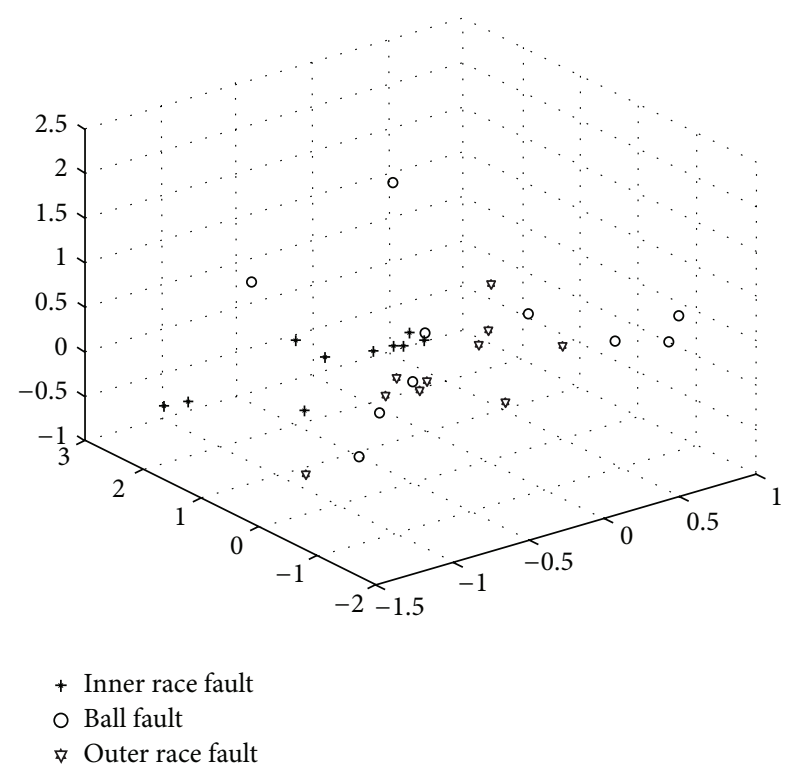

FIGURE 6: The result of the signal not purified by optimized morphological filter and use of the LTSA to extract the original features.

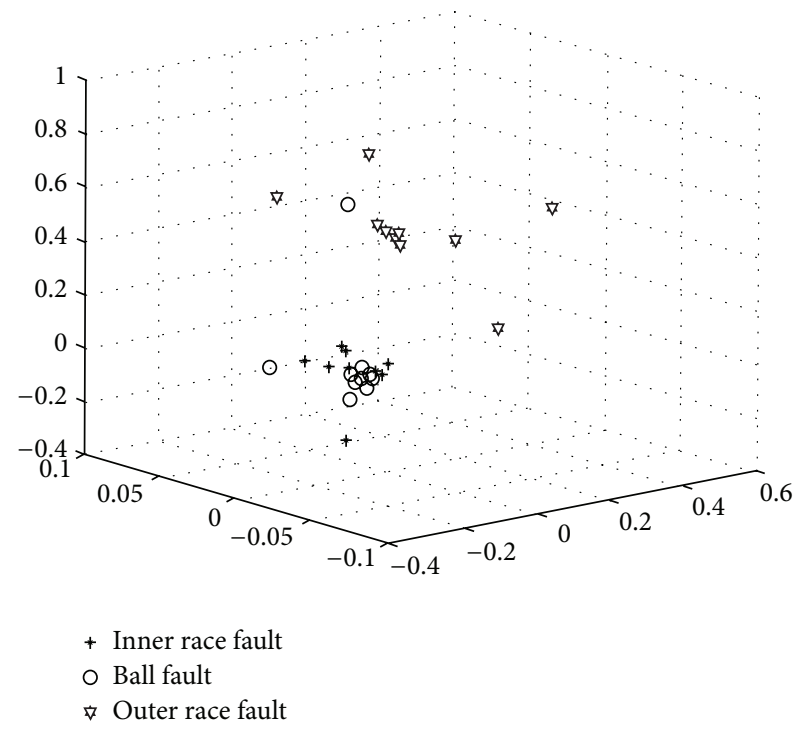

FIGURE 7: The result of the signal purified by optimized morphological filter and use of the PCA to extract the original features.

(4) Despite the different comparisons we can see that the proposed method makes good use of the advantage of all parts and together to obtain a better recognition accuracy and efficiency.

\section{Conflict of Interests}

The authors declare that there is no conflict of interests regarding to the publication of this paper.

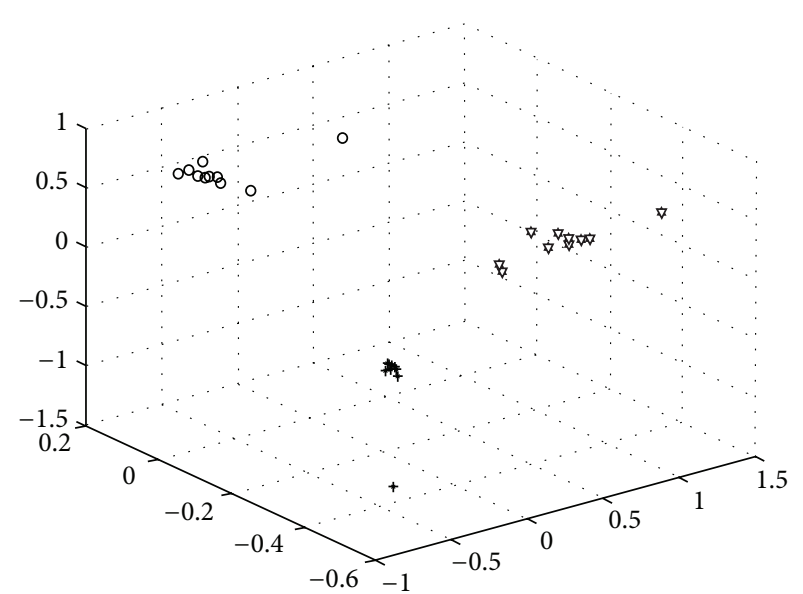

$$
\begin{aligned}
& + \text { Inner race fault } \\
& \text { ○ Ball fault } \\
& \star \text { Outer race fault }
\end{aligned}
$$

FIGURE 8: The result of the signal purified by optimized morphological filter and use of the LTSA to extract the original features.

\section{Acknowledgments}

This research is supported by the National Natural Science Foundation of China (nos. 51405047, 51275546, and 51405048), Natural Science Foundation Project of CQ cstc2013jcyjA70012. China Postdoctoral Science Foundation funded this research, Project no. 2014M552316. The authors are grateful to the anonymous reviewers for their helpful comments and constructive suggestions.

\section{References}

[1] S. Du, J. Lv, and L. Xi, "Degradation process prediction for rotational machinery based on hybrid intelligent model," Robotics and Computer-Integrated Manufacturing, vol. 28, no. 2, pp. 190207, 2012.

[2] R. Yan, R. X. Gao, and X. Chen, "Wavelets for fault diagnosis of rotary machines: a review with applications," Signal Processing, vol. 96, pp. 1-15, 2014.

[3] Y. Qin, B. Tang, and J. Wang, "Higher-density dyadic wavelet transform and its application," Mechanical Systems and Signal Processing, vol. 24, no. 3, pp. 823-834, 2010.

[4] S. Du and J. Lv, "Minimal Euclidean distance chart based on support vector regression for monitoring mean shifts of auto-correlated processes," International Journal of Production Economics, vol. 141, no. 1, pp. 377-387, 2013.

[5] S. Nishida, M. Nakamura, A. Ikeda, and H. Shibasaki, "Signal separation of background EEG and spike by using morphological filter," Medical Engineering and Physics, vol. 21, no. 9, pp. 601-608, 1999.

[6] S. Du, L. Xi, J. Yu, and J. Sun, "Online intelligent monitoring and diagnosis of aircraft horizontal stabilizer assemble processes," International Journal of Advanced Manufacturing Technology, vol. 50, no. 1-4, pp. 377-389, 2010.

[7] Z.-Y. Zhang and H.-Y. Zha, "Principal manifolds and nonlinear dimensionality reduction via tangent space alignment," Journal of Shanghai University, vol. 8, no. 4, pp. 406-424, 2004. 
[8] X. Huo and A. K. Smith, "Matrix perturbation analysis of local tangent space alignment," Linear Algebra and its Applications, vol. 430, no. 2-3, pp. 732-746, 2009.

[9] T. Zhang, J. Yang, D. Zhao, and X. Ge, "Linear local tangent space alignment and application to face recognition," Neurocomputing, vol. 70, no. 7-9, pp. 1547-1553, 2007.

[10] S. Du, C. Liu, and L. Xi, "A selective multiclass support vector machine ensemble classifier for engineering surface classification using high definition metrology," Journal of Manufacturing Science and Engineering, vol. 137, no. 1, Article ID 011003, 2015.

[11] N. G. Nikolaou and I. A. Antoniadis, "Demodulation of vibration signals generated by defects in rolling element bearings using complex shifted Morlet wavelets," Mechanical Systems and Signal Processing, vol. 16, no. 4, pp. 677-694, 2002.

[12] P. Maragos and R. W. Schafer, "Morphological filters part I: their set-theoretic analysis and relations to linear shift-invariant filters," IEEE Transactions on Acoustics, Speech, and Signal Processing, vol. 35, no. 8, pp. 1153-1169, 1987.

[13] W. He, Z. Jiang, and Q. Qin, "A joint adaptive wavelet filter and morphological signal processing method for weak mechanical impulse extraction," Journal of Mechanical Science and Technology, vol. 24, no. 8, pp. 1709-1716, 2010.

[14] S. Du, D. Huang, and J. Lv, "Recognition of concurrent control chart patterns using wavelet transform decomposition and multiclass support vector machines," Computers \& Industrial Engineering, vol. 66, no. 4, pp. 683-695, 2013.

[15] S. Du, J. Lv, and L. Xi, “On-line classifying process mean shifts in multivariate control charts based on multiclass support vector machines," International Journal of Production Research, vol. 50, no. 22, pp. 6288-6310, 2012.

[16] “Case Western Reserve University bearing data center," 2009, http://www.case.edu/source/. 

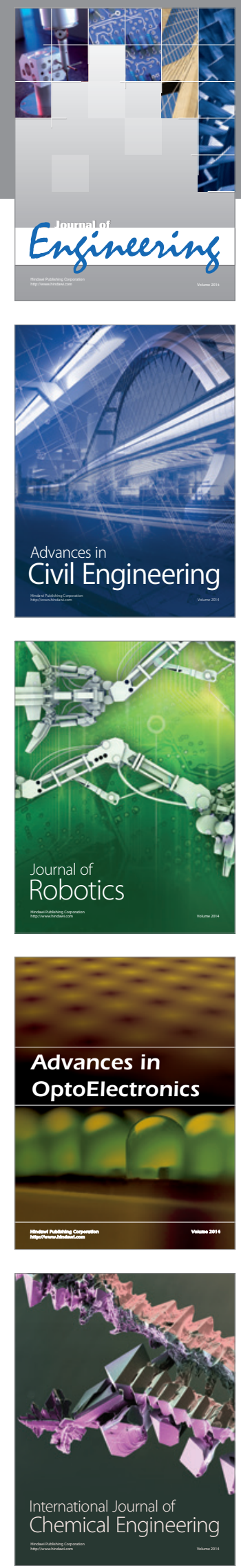

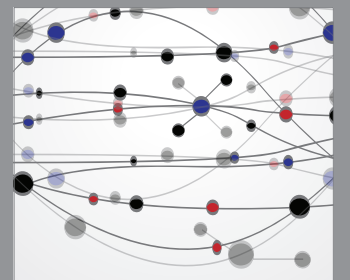

The Scientific World Journal
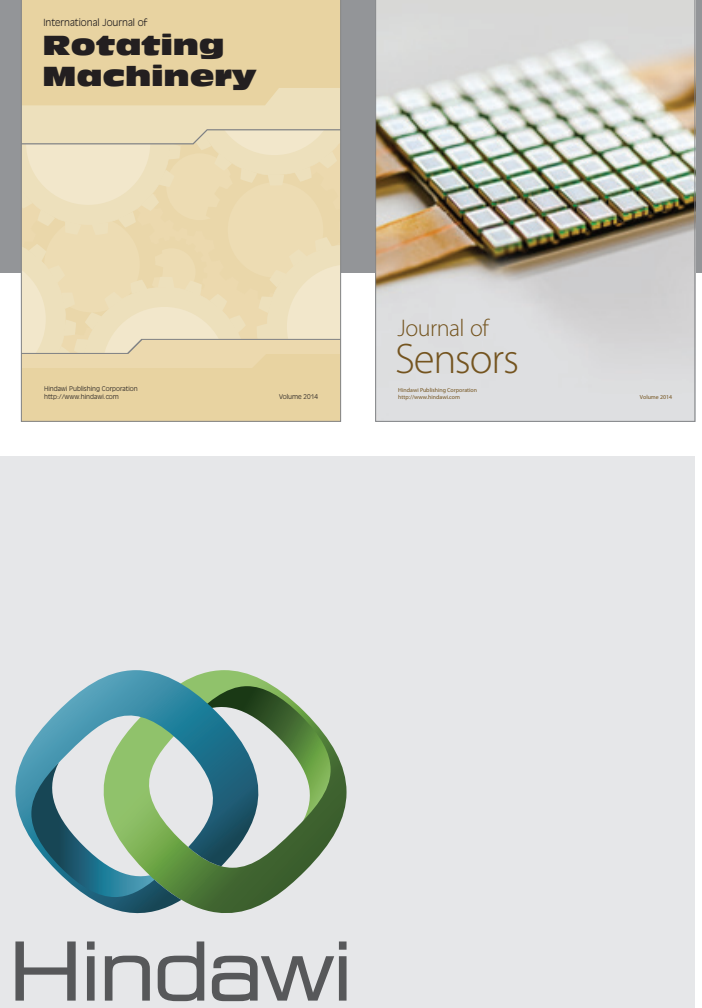

Submit your manuscripts at http://www.hindawi.com
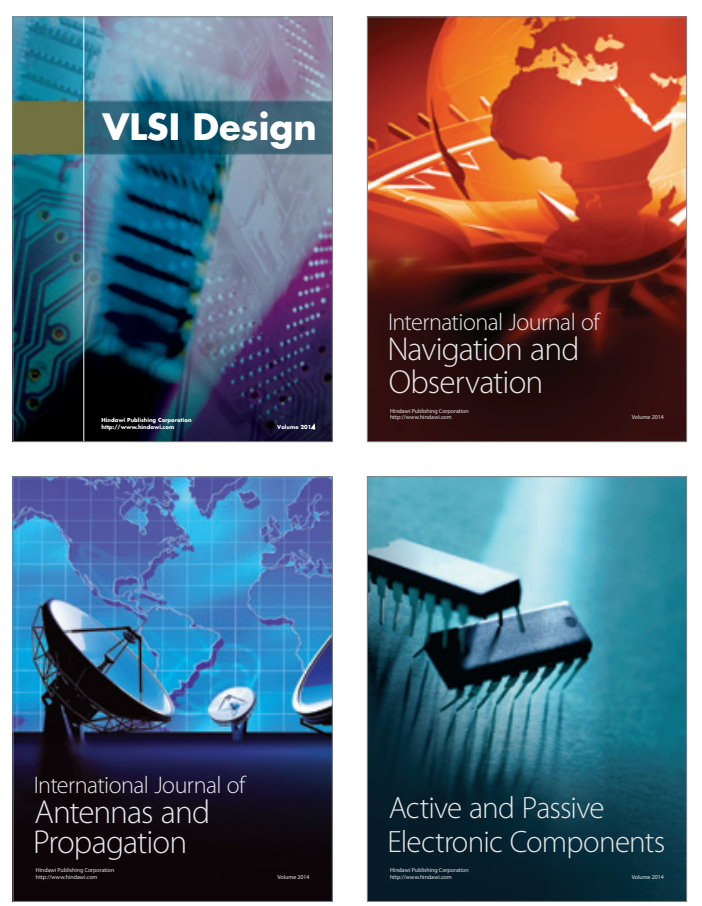
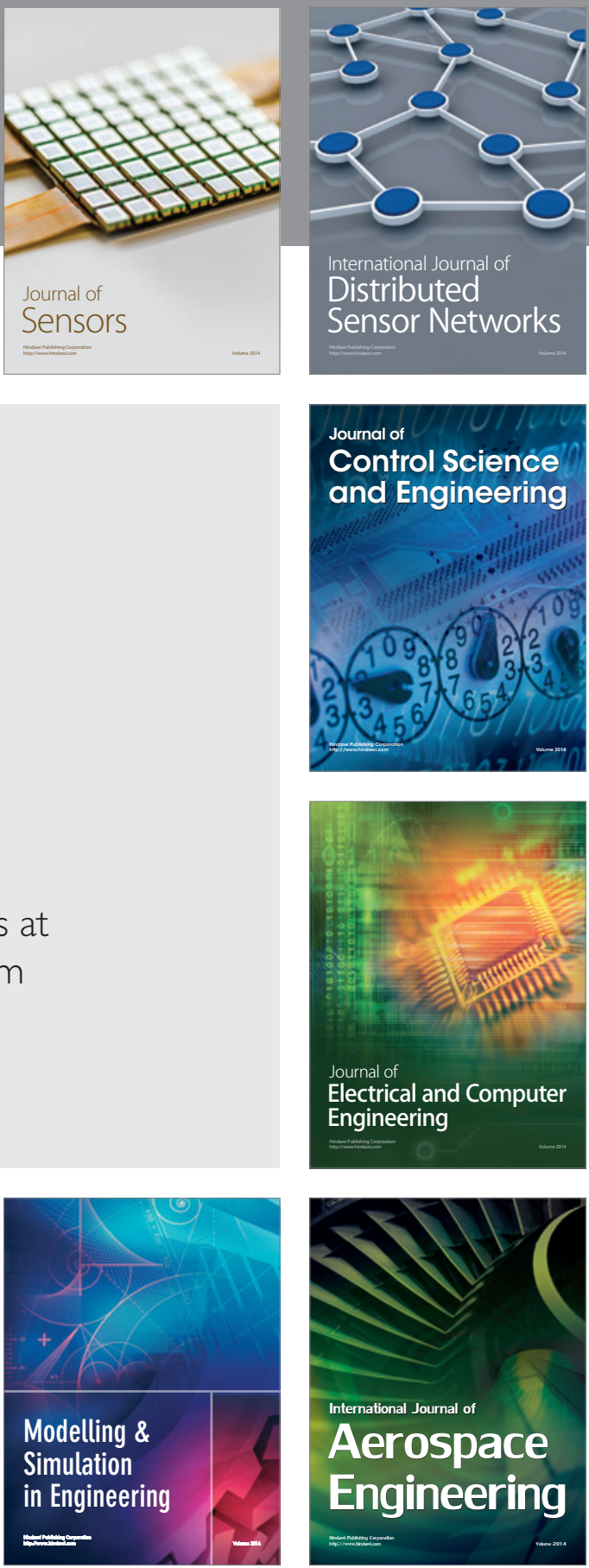

Journal of

Control Science

and Engineering
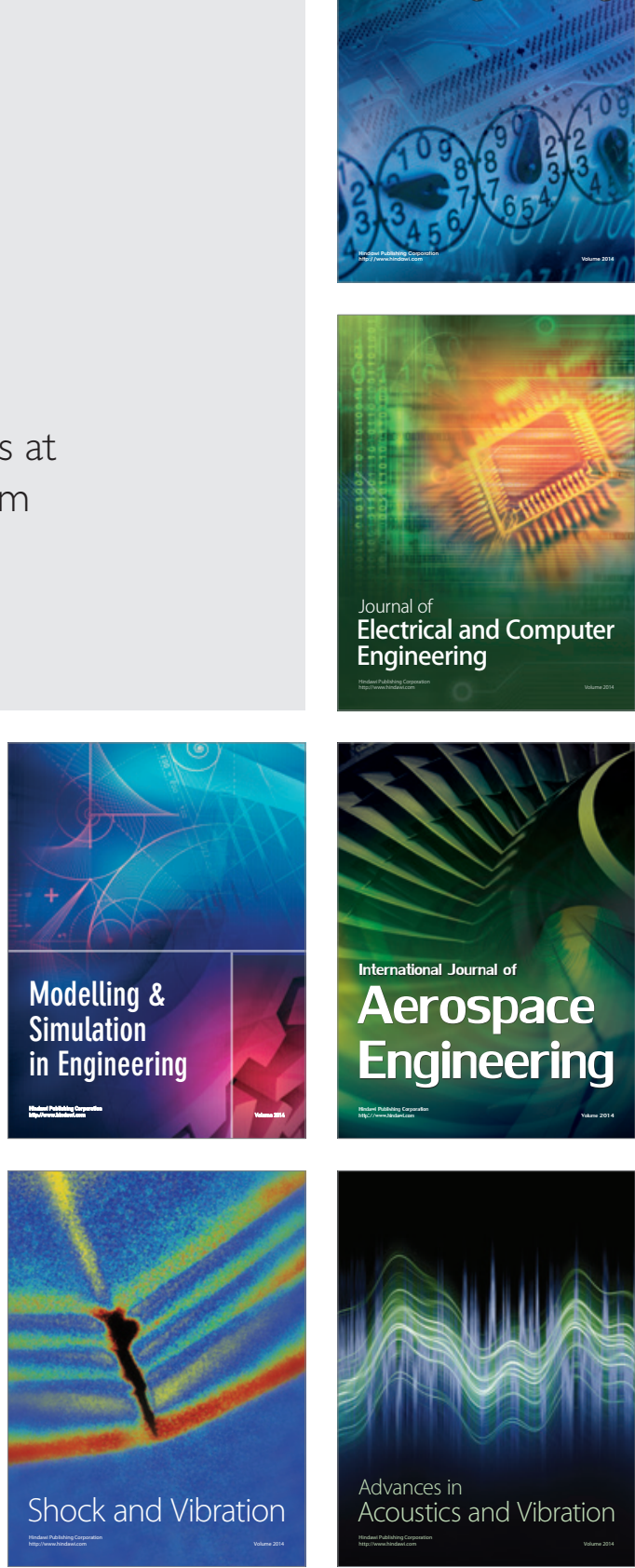